\title{
THE NRAO VLA D-ARRAY SKY SURVEY (NVSS)
}

W. D. COTTON, J. J. CONDON AND Q. F. YIN

National Radio Astronomy Observatory ${ }^{\ddagger}$

520 Edgemont Rd, Charlottesville, VA 22903-2475, USA

R. A. PERLEY

National Radio Astronomy Observatory

Post Office Box 0, Socorro, NM 87801-0387, USA

AND

J. J. BRODERICK

Virginia Tech $\S$

Physics Department, Virginia Tech, Blacksburg, VA 24061, $U S A$

\section{Introduction}

Early large scale radio surveys of the sky were made with instruments with poor imaging quality and were limited to measuring positions and brightnesses of discrete sources. In recent decades radio interferometric arrays have dramatically increased their speed, sensitivity and ability to image the sky and several large scale radio surveys are currently being made with imaging instruments. One of these surveys is discussed in this paper.

\section{Survey Design and Goals}

The National Radio Astronomy Observatory (NRAO) is using the Very Large Array (VLA) in its most compact configuration, the " $D$ " array, to image the sky north of declination $-40^{\circ}$ at a frequency of $1.4 \mathrm{GHz}$ (or 20 $\mathrm{cm}$. wavelength). This survey will produce $23264^{\circ} \times 4^{\circ}$ images in Stokes I, $\mathrm{Q}$, and U (total intensity and linear polarization) with a resolution of $45^{\text {" }}$ FWHM. There will also be corresponding calibrated and edited interferometer data and a list of approximately $2,000,000$ discrete sources derived

†The National Radio Astronomy Observatory is a facility of the National Science Foundation operated under cooperative agreement by Associated Universities, Inc.

${ }^{\S}$ Research is supported in part by NSF grant AST-93-20547 
from these images. The survey images will have a nearly uniform 5 sigma detection limit of approximately $2.5 \mathrm{mJy} /$ beam which corresponds to 0.75 $\mathrm{K}$ for sources approximately the same size as the synthesized beam. The rms position uncertainties range from $<1$ " for $\mathrm{S}>10 \mathrm{mJy}$ to approximately 5 " at $\mathrm{S}=2.5 \mathrm{mJy}$. The RMS noise in the linearly polarized images is typically $0.3 \mathrm{mJy} /$ beam allowing " $1 \sigma$ detections" of $12 \%$ polarized sources even at the survey limit. Observations in the far north and south where the array is foreshortened in the north-south direction use the the hybrid "DnC" array to maintain the circularity of the synthesized beam. Due to the interferometric nature of the instrument structure on scales larger than about $10^{\prime}$ 'will have a reduced response. It is therefore expected that the majority of sources detected will be extragalactic. The design of this survey is discussed in more detail in [2].

\section{Current Status}

The observations are being done in three sessions to allow the observations to be done at night. The first of these sessions began in 1993 September, the second in 1995 January and the third will be in the summer of 1996 . Approximately two thirds of the sky has been observed although some pointings will have to be repeated due to strong interference. All of the individual pointing data have been imaged from the first session as well as the DnC observations from the second sessions. The final $4^{\circ} \times 4^{\circ}$ images from the first session are available as well as a source list derived from them.

\section{Availability of Results}

The principle data products, images, interferometer data and source list, are being released as they become available. The source list is updated as new images become available. The products of the NVSS are available via anonymous $\mathrm{ftp}$ (ftp://nvss.nrao.edu/pub/nvss). Images are available as gzipped FITS files and the source list is kept in the form of a FITS binary table [3]. A browser (http://info.cv.nrao.edu/NVSS/NVSS.html) is available to search the source list. More information is available on the NVSS home page (http://info.cv.nrao.edu/ jcondon/nvss.html).

\section{Comparison with UGC galaxies}

The UGC catalog of galaxies [5] contains approximately $10^{4}$ galaxies with blue diameter $\theta \geq 1$ arc min and declination $\delta \geq-2.5^{\circ}$. The positions of the galaxies in the original catalog are only good to $\approx 1^{\prime}$ which is insufficiently accurate for reliable identification with sources from the NVSS. A subset of the brighter of these galaxies $(\mathrm{m}<14.5)$ have more accurate positions 


\section{NVSS Detections of UGC Galaxies}

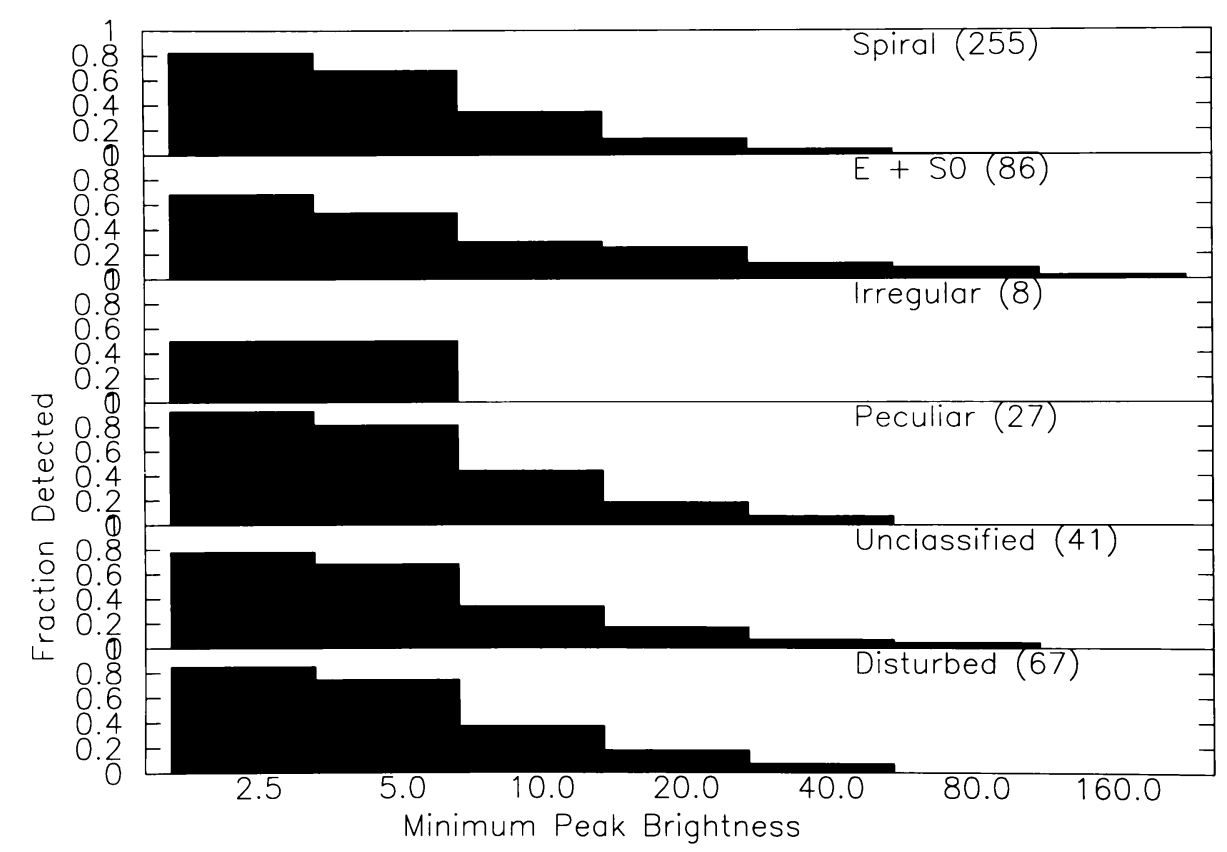

Figure 1. This figure shows the fraction of UGC galaxies with magnitudes between 12.5 and 14.5 detected by the NVSS as a function of limiting peak brightness. Each plot is labeled with the galaxy classification with the number of galaxies in the sample in parentheses. The total number of galaxies is 417 , The category "Disturbed" includes those of all morphological types which were indicated as disturbed in some way in the UGC.

$(\approx 4 ")$ measured by [4]. The fields of these galaxies with Right ascensions between $0^{h}$ and $10^{h}$ and in the magnitude range $12.5-14.5$ were searched for NVSS sources within 15" of the optical positions. Figure 1 shows the fraction of these galaxies detected by the NVSS as function of limiting peak radio brightness for a variety of morphological categories and for all galaxies marked in the UGC as "disturbed", "distorted", "eruptive", or "peculiar". The relatively small number of objects in Figure 1 is due to the low galactic latitude of the region of overlap between the NVSS and the UGC.

The false detection rate of galaxies within 15 " of the radio position should be fairly small. This assertion is supported by the results of a test in which only 3 of 444 galaxies were found "coincident" with radio sources when all the optical positions were given a 5 ' offset. The high fraction of detections of all categories of galaxies suggests that these relatively nearby 
galaxies have detectable levels of radio emission - even the ellipticals and lenticulars which are generally gas poor (cf. [7]). The high detection rate of "disturbed" galaxies also suggests that the peculiarities associated with these galaxies may be related to star formation activity. A relatively high fraction $(\approx 60 \%)$ of these sources are resolved by the NVSS with sizes larger than a few tens of arc seconds. This is to be compared with only $29 \%$ of the sources in a sample of 18,000 sources at high galactic latitude appearing resolved.

A comparison of NVSS flux densities with the $60 \mu$ flux densities of IRAS indicate that the majority of spirals and "disturbed" galaxies $(72 \%$ and $76 \%$ respectively) have ratios consistent with star formation. However, only a relatively small fraction $(14 \%$ - all $\mathrm{S} 0)$ of the $\mathrm{E} / \mathrm{S} 0$ galaxies have radio/IR flux ratios consistent with star formation.

The high detection rate of spiral galaxies $(210 / 255$ or $82 \%)$ is consistent with low-resolution $1.4 \mathrm{GHz}$ VLA images of the brightest spiral and irregular galaxies [1]. The only VLA surveys of bright $\mathrm{E} / \mathrm{S} 0$ galaxies [6], [8], were made with $\theta \approx 5^{\prime \prime}$ resolution at $\nu=4.86 \mathrm{GHz}$, so they didn't have the surface brightness sensitivity to detect weak, extended regions. Only $52 / 198(25 \%) \mathrm{E} / \mathrm{S} 0$ galaxies were detected by [8] with a flux density limit of $0.5 \mathrm{mJy}$ whereas we detect 59 of $86(69 \%)$ above $2.5 \mathrm{mJy}$.

The median face on surface brightness of spiral galaxies, $\langle\mathrm{T}\rangle \approx 1 \mathrm{~K}$ at $1.4 \mathrm{GHz}$, is reachable for the first time by the NVSS. The NVSS will eventually cover the entire region of the UGC allowing a comparison with the nearly 3800 galaxies with accurate optical positions.

\section{References}

[1] Condon, J. J. and Cotton, W. D. and Greisen, E. W. and Yin, Q. F. and Perley R. A. and Broderick, J. J., "The NRAO VLA sky survey. I. goals, methods and first results", in preparation, 1996,

[2] Condon, J. J, "A $1.4 \mathrm{GHz}$ atlas of Spiral Galaxies with $\mathrm{B}_{T} \leq+12$ and $\delta \geq-45^{\circ}$ ", Astrophys. J. Suppl., 1987, 65, 485

[3] Cotton, W. D. and Tody, D. and Pence, W. D., "Binary table extension to FITS", Astron. Astrophys. Suppl., 1995, 113, 159

[4] Dressel, L. L. and Condon, J. J, "Accurate Optical Positions of Bright Galaxies", Astrophys. J. Suppl., 1976, 31, 187

[5] Nilson, P., Uppsala General Catalog of Galaxies, Uppsala Astronomical Observatory, Uppsala, Sweden, 1973, 456

[6] Sadler, E. M. and Jenkins, C. R. and Kotanyi, C. G., "Low-Luminosity radio sources in early-type galaxies", Mon. Not. Roy, Astron Soc., 1989, 240, 591

[7] Wrobel, J. M. and Heeschen, D. S., "Current star formation as the origin of kiloparsecscale radio sources in nearby E/S0 Galaxies", A strophys. J., 1988, 335, 677

[8] Wrobel, J. M. and Heeschen, D. S., "Radio Continuum Sources in nearby and Bright E/S0 galaxies: active nuclei versus star formation", Astron. J., 1991, 101, 148 